be recommended to those individuals that achieve a score under $50 \%$. The software that will be used is Question Writer professional 4, which is licenced to SIFO.

Results In order to be able to present the results at the EAHP congress in Paris, the questionnaire will be available online, to be answered during January-February 2012. The SIFO will dispatch the survey to some 2000 SSN pharmacists via email making use of the society's mailing list. The results will be presented in a poster and the final situation about the general skills of Italian SSN pharmacists regarding leadership will be presented by area as specified in the RPS framework. These results will provide an overview of the knowledge of Italian pharmacists and SIFO intends to arrange specific training courses in follow-up and to encourage the participants who do not get good scores to engage in autonomous training.

Conclusions Pharmacists' awareness of leadership and management, acquired by completing the questionnaire and being awarded an individual skills level, will be an incentive for the SIFO and other professionals to undertake the necessary corrective activities, such as education and specific training. We would like to start a selfawareness path regarding the importance of leadership competences in the personal CVs of pharmacists working in the Italian SSN. The ultimate aims of improving leadership skills are improved costeffectiveness, better quality services, and risk reduction in patients who benefit from the SSN services. The questionnaire will be available in English, for it to be used within the EAHP and other European scientific societies.

No conflict of interest.

\section{BEA-003 OPTIMIZATION OF TREATMENT SAFETY AT THE IN- AND OUT-PATIENT INTERFACE IN NEUROSURGICAL CARE}

doi:10.1136/ejhpharm-2013-000276.614

P Kantelhardt, A Giese, SR Kantelhardt. Universitätsmedizin Mainz, Neurochirurgie, Mainz, Germany

Background At admission and discharge to/from hospital information concerning the correct medicines has to be transferred between health professionals. If this information is incomplete or lost, the correct medicines for patients are at risk. We therefore analysed adverse events at the in-/out-patient interface in order to optimise the medical treatment of patients at these critical steps.

Purpose To optimise the medical treatment of patients at admission and discharge to/from hospital.

Materials and Methods The prescription and resulting administration of medicines of all patients who underwent spinal stabilisation surgery in our clinic in the year of 2011 were recorded retrospectively. We analysed the resulting dataset in terms of frequency and severity of medicines errors.

Results 147 datasets were included, while only 144 of these contained complete information concerning post-discharge medicines.

The medicines taken before admission to the hospital were not documented correctly in $16 \%$ of the admission reports. Complete transfer of the previously taken medicines to drugs listed by the hospital pharmacy was missing in $72 \%$. Both these factors frequently led to incomplete continuation of the medicines taken previously (before admission). Uncertainty concerning the listed drugs [the medicines prescribed for use in hospital] was identified as the main reason for this problem.

At discharge the prescribed medicines did not match the medicines taken before admission in $78 \%$. An indication for this change was however only documented in $9 \%$.

Missing documentation of the medicines taken before admission and an unconsidered transposition of the drugs listed in the hospital pharmacy to the discharge information were identified as the most common risk factors.
Furthermore in $37 \%(n=41$ who received anticoagulation treatment) and in $67 \%(n=9$ who received metformin) these treatments, which were paused preoperatively, were not resumed postoperatively.

So in order to optimise treatment at the in-/out-patient interface a number of processes were modified:

- At admission - the medicines history is now taken by a clinical pharmacist, who was employed for this purpose instead of a physician. The pharmacist is informed via the hospital administration software (SAP) or the admissions management system when a new patient is admitted.

- The hospital pharmacist transfers previously taken medicines to the listed drugs.

- A new admission sheet was designed standardising the recording of medicines history and transfer to listed drugs. This sheet provides all the necessary information concerning the drugs taken previously to physicians and nurses in a standardised form throughout hospitalisation and when composing the discharge information.

These measures allow continuous administration of the medicines taken before admission over the in-/out-patient interfaces and readministration after the perioperative period of suspended drugs like anticoagulants and metformin. The clinical pharmacist furthermore cheques the medicines in stock of each unit of the clinic and orders any new or special drugs form the hospital pharmacy. During hospitalisation the physicians consult the clinical pharmacist on specific medicines issues.

Conclusions In order to achieve a high level of medicines safety physicians, nurses and clinical pharmacists have to cooperate closely and frequently. Each step in the medicines process should be performed by the specialist most suited for this task. The medicines process has to be standardised and transparent, so that each group involved (nurses, physicians, clinical pharmacists) knows at any time where to find the required information. In order to achieve this, the neurosurgical department now employs our 'own' clinical pharmacist. A final review of the measures taken and the overall quality of medicines at the in-/out-clinic interface is scheduled for 2013

No conflict of interest.

\section{BEA-004 TAKING A LEAD IN WARD PATIENT SAFETY}

doi:10.1136/ejhpharm-2013-000276.615

M Ulgey, E Guner. Konya Numune State Hospital, Pharmacy, Konya

Background The pharmacist workforce is limited in terms of patient safety due to the 'one pharmacist to every one hundred beds' rule in Turkish state hospitals. Our hospital is being rebuilt, and having fewer patients in wards has resulted in all departments working under capacity for a certain period.

Purpose To take advantage of this unique situation that allowed pharmacists to raise the standards of patient safety by using the extra time and workforce granted; and also to prove that good leadership in pharmacy care can result in better patient health.

Materials and Methods Pharmacists were encouraged to appraise the clinical skills of their department, determine the level of the need of ward patients for better patient safety and judge the resources currently available for implementation, before considering the potential sources of collaboration with other health professionals. 'Rx Media Pharma' software was used for gaining detailed results on patient chart evaluations. All documentation was performed online with 'Google Docs', allowing participants to share and make changes online directly with selected health professionals.

Results For 23 working days, 200 patient charts were reviewed. The average number of drugs used was 7.6 and the drug-drug interactions identified were 2.02 per patient. The importance of the interactions was evaluated in 3 levels; major (42.82\%), moderate 
$(51.23 \%)$ and minor (5.94\%). The numbers of recommendations regarding the drug-drug interactions spotted were: 31 therapeutic exchange (7.67\%), 88 dosage recommendation (21.78\%), 4 adding drug to the treatment $(0.99 \%), 99$ proposal to withdraw a particular drug (24.50\%), 182 monitoring (45.04\%). Total number of food-drug interactions was 286 (1.43 per patient), with 118 instances of intravenous incompatibility warnings made to the ward nurses ( 0.59 per patient). 62 inappropriate drug dosages ( 0.31 per patient) and 3 drugs containing the same active substances in different formulations ( 0.015 per patient) were reported to the prescribing physicians. In 124 cases (0.62 per patient), pharmacists requested information about the use of the drugs prescribed for treatment.

Upon discussion of the results, the physicians provided feedback and acted according to three options: (9.14\%) the physician didn't agree (they believed the situation didn't require an intervention), $(58.53 \%)$ the physician felt it was sufficient to monitor the patient's status, considering the suggested change in treatment, (32.31\%) the physician agreed to a change in the patient's treatment, applying the recommendation pharmacists made.

Conclusions Our pharmacy department discovered that continuous service at this level of quality is needed for ward patients. Similar studies should be encouraged by health care leaders in Turkey to improve hospital care.

No conflict of interest.

\section{BEA-005 UNLICENSED AND OFF-LABEL DRUG PRESCRIPTION AT DISCHARGE FROM A SWISS CHILDREN'S HOSPITAL}

doi:10.1136/ejhpharm-2013-000276.616

${ }^{1} \mathrm{C}$ Zaugg, ${ }^{1} \mathrm{~J}$ Behringer, ${ }^{2} \mathrm{M}$ Walther, ${ }^{2 \mathrm{M} \text { Köhler. }}$ 'Spitalapotheke, Kantonsspital Aarau; ${ }^{2}$ Klinik für Kinder und Jugendliche, Kantonsspital Aarau

\section{Introduction.}

For children, many drugs are used without marketing authorization ("unlicensed", e.g. imported drugs, drugs prepared by a pharmacy) or outside the terms of marketing authorization ("off-label"). In Switzerland, around half of all prescriptions for paediatric inpatients were either off-label or unlicensed [1].

Purpose To determine the proportion of unlicensed and off-label prescriptions at discharge, which has not been investigated previously, and the proportion of parents informed about such a prescription.

Materials and Methods Prospective study including all discharge prescriptions of inpatients over a two-month period at the Children's Hospital of Aarau. Exclusion criteria: hospitalisation for chemotherapy only, age over 18, re-entry during study period, no informed consent of parents. At discharge parents were asked to fill in a questionnaire about the information they got on discharge medicines as well as about their satisfaction with this information. This questionnaire was available in German, French, Croatian, Turkish, Albanian, Spanish and English.

Results During the study period 503 children were discharged, 231 children could be included. Discharge prescriptions were written for 140 children (61\%). A total of 227 drugs were prescribed, especially anti-inflammatory/analgesic, anti-asthmatic and anti-infective drugs. $38.5 \%$ of all prescriptions were off-label, regarding dosage in $51 \%$, age in $40 \%$ and indication in $9 \%$ of all cases. Only $0.5 \%$ of drugs were unlicensed. Discharge questionnaires were returned by 103 of 140 children. Most parents (>80\%) were informed about purpose, dosage and use of the drugs for their child, and satisfied with obtained information, but only $9 \%$ of parents getting an off-label/unlicensed prescription for their child were informed about the off-label/unlicensed use.

Conclusions There is a high percentage of drugs prescribed offlabel at hospital discharge. Most drugs are well known substances and regularly prescribed for children. This emphasises the need to update marketing information for older substances, or the need for a national database for drug use and dosage in children.

\section{Reference}

1. Paolo, E et al, 2006. Swiss. Med. WKLY. 136, 218-222.

No conflict of interest.

\section{BEA-006 USING LEADERSHIP TO TURN DEFEAT INTO SUCCESS}

doi:10.1136/ejhpharm-2013-000276.617

\section{A Forsstrom. Hospital Pharmacy at the University Hospital in Uppsala}

Background I was a manager at the Hospital Pharmacy at the University Hospital in Uppsala. The hospital has about 1100 beds. The hospital pharmacy employs a staff of about 75. Production consisted of both chemotherapy unit preparations and other sterile preparations. This Friday afternoon in November, I was summoned to the hospital's Chief Medical Officer. Once there, I receive complaints on the service from our production unit for Chemotherapy Preparations. Orders were often delivered too late - No notice of delays - Lack of communication between the hospital staff and the staff at the production unit.

Purpose To show how we improved the service from the Centralised Chemotherapy IV Preparation Unit.

Materials and Methods It was important to use my leadership to see something positive in what happened. It was not our skills that were complained about, but our service, which made it all easier. My point was, we are competent and skilled, now we have to improve our service. Together with the staff we decided:

- The complaint in terms of communication was true. Action: Hospital staff were invited.

- Tuesdays and Thursdays we had information for the staff, and we were always late. Action: We changed to providing more written information and just assembled the staff one Tuesday and Thursday every month.

- We did not answer the phone, or call and notify the delay. Action: We extended the staff in the morning by a technician who could answer the phone. Then we agreed to measure the delays. We completed a document in which we recorded when orders come in, if they were complete or if it needed a phone contact before they would be prepared. Then we documented when the preparations were ready. We decided to measure for 4 weeks in December. This measurement has since then been performed every year. We could pretty quickly see that we often received orders late and they were not complete.

Delivery of chemotherapy from Centralised Chemotherapy IV Preparation Unit at Uppsala University Hospital. Data for each year show the fraction of preparations delivered on time, fraction delivered after the requested time and the average time delay.

\section{Abstrtact BEA-006 Table 1}

\begin{tabular}{llllllll}
\hline Year & $\mathbf{2 0 0 5}$ & $\mathbf{2 0 0 6}$ & $\mathbf{2 0 0 7}$ & $\mathbf{2 0 0 8}$ & $\mathbf{2 0 0 9}$ & $\mathbf{2 0 1 0}$ & $\mathbf{2 0 1 1}$ \\
\hline Percentage delivered on time & $90 \%$ & $96 \%$ & $94 \%$ & $93 \%$ & $98 \%$ & $95 \%$ & $99 \%$ \\
Time delay & $19 \mathrm{~min}$ & $32 \mathrm{~min}$ & $16 \mathrm{~min}$ & $18 \mathrm{~min}$ & $32 \mathrm{~min}$ & $28 \mathrm{~min}$ & $36 \mathrm{~min}$ \\
\hline
\end{tabular}

These results were discussed later with the hospital staff and together we were able to improve the ordering schedule and we improved service with the deliveries. More preparations could be delivered on time.

Conclusions Inform your manager and staff. Take your time and plan how to handle the situation. Try to find out what you and your staff can learn. Try to understand the cause of the complaint and motivate staff for change. Try to measure in order to have a base for discussion and change. Good management is extra important for to support your staff when you and your staff are questioned.

No conflict of interest. 